\title{
Indocyanine Green Angiography Findings in Initial Acute Pretreatment Vogt-Koyanagi-Harada Disease in Japanese Patients
}

\author{
Masaru Miyanaga ${ }^{1,2}$, Tatsushi Kawaguchi ${ }^{1}$, Kazunori Miyata ${ }^{2}$, Shintaro Horie $^{1}$, \\ Manabu Mochizuki ${ }^{1}$, and Carl P. Herbort ${ }^{1,3,4}$ \\ ${ }^{1}$ Department of Ophthalmology and Visual Science, Tokyo Medical and Dental University \\ Graduate School of Medical and Dental Sciences, Tokyo, Japan; ${ }^{2}$ Miyata Eye Hospital, \\ Miyazaki, Japan; ${ }^{3}$ Inflammatory and Retinal Eye Diseases, Centre for Ophthalmic Specialized \\ Care, Lausanne, Switzerland; ${ }^{4}$ University of Lausanne, Lausanne, Switzerland
}

\begin{abstract}
Purpose: Indocyanine green angiography (IA) is a highly sensitive method to evaluate choroidal inflammatory lesions. We present standardized IA findings of initial acute Vogt-Koyanagi-Harada (VKH) disease in Japanese patients before therapeutical intervention.

Methods: Medical records of patients with VKH disease at Tokyo Medical and Dental University Hospital and Miyata Eye Hospital were retrospectively analyzed. We analyzed six IA signs: choroidal perfusion inhomogeneity, early hyperfluorescent stromal vessels, hypofluorescent dark dots (HDDs), fuzzy or lost pattern of large stromal vessels, disc hyperfluorescence, and diffuse late choroidal hyperfluorescence.

Results: Ten patients from the two hospitals were studied. The most constant findings present in all eyes were early hyperfluorescent stromal vessels, HDDs, and either fuzzy or lost pattern of large stromal vessels. Disc hyperfluorescence was present in 18 eyes. Choroidal perfusion inhomogeneity was seen in six patients, and diffuse late choroidal hyperfluorescence was seen to a certain degree in all eyes.

Conclusions: Four of the analyzed signs, including early hyperfluorescent stromal vessels, HDDs, fuzzy or lost pattern of large stromal vessels, and disc hyperfluorescence were consistent findings in Japanese VKH patients. Because the primary lesion is situated in the choroid, IA is the method of choice to monitor disease activity in VKH disease. Jpn J Ophthalmol 2010;54:377-382 @ Japanese Ophthalmological Society 2010
\end{abstract}

Keywords: choroidal inflammation, indocyanine green angiography, Japanese patients, Vogt-KoyanagiHarada disease

\section{Introduction}

Vogt-Koyanagi-Harada (VKH) disease is a nontraumatic, bilateral, granulomatous panuveitis accompanied by a set

\footnotetext{
Received: January 12, 2010 / Accepted: April 26, 2010

Correspondence and reprint requests to: Carl P. Herbort, Inflammatory and Retinal Eye Disease, Centre for Ophthalmic Specialized Care, 6 Rue de la Grotte, CH-1003, Lausanne, Switzerland

e-mail: carl.herb@bluewin.ch
}

of systemic symptoms including meningismus, tinnitus, alopecia, poliosis, and vitiligo. ${ }^{1,2}$ The most commonly observed ocular involvements are iridocyclitis, multifocal areas of serous retinal detachment, and redness and swelling of the optic disc. VKH disease is considered to be an autoimmune disease, and specific targets in the posterior segment are stromal choroidal melanocytes. There is increasing evidence that the disease mechanism is an autoimmune T-helper type 1 reaction directed against proteins related to stromal choroidal melanocytes. ${ }^{3-5}$ Therefore, the initial 
posterior ocular inflammatory events in VKH disease selectively involve the choroid.

Indocyanine green angiography (IA) is an established and highly sensitive method for the evaluation of choroidal inflammatory lesions. ${ }^{6}$ For posterior uveitis with choroidal involvement such as VKH disease, IA has been shown to be the most sensitive method to assess and follow choroidal inflammation. ${ }^{7}$ The fluorescence of the indocyanine green (ICG) dye occurs in the near-infrared wavelengths and can be detected through the retinal pigment epithelium, allowing imaging access to the choroidal vascular structures and the stroma. ${ }^{8}$ Since IA has made detection of choroidal lesions possible, several reports have been published on the choroidal involvement in VKH disease. ${ }^{9-14}$ Several IA signs have been presented in different series, but their practical value for clinical use has not been clearly determined as the patients analyzed were at different stages of disease and therapy. Diverse IA protocols and methods have been used to analyze VKH patients at more or less advanced stages of disease to describe findings in VKH patients; thus, systematization of IA signs has not been possible. Systematization of IA signs in VKH disease has been undertaken recently, in which a set of IA signs consistently found in $\mathrm{VKH}$ patients was put forward, which proved very useful for the follow-up of the choroidal inflammation. ${ }^{13,14}$ Submaximal doses of inflammation suppressive therapy can suppress a clinically apparent disease but not the localized choroidal inflammation that only IA can detect. ${ }^{15}$ This explains the propensity for sunset glow fundus in seemingly controlled disease.

In the current study, we retrospectively analyzed IA findings using a standardized IA protocol ${ }^{6}$ in a homogeneous group of Japanese patients presenting an initial acute episode of VKH disease at presentation before therapeutical intervention to determine whether previously proposed IA signs ${ }^{13,14}$ are sufficiently constant, easily recordable, and quantifiable to be useful for the evaluation and monitoring of choroidal inflammation in Japanese patients.

\section{Materials and Methods}

Medical records of VKH patients that had a standard IA performed before treatment either at Tokyo Medical and Dental University Hospital Ophthalmology Department or at Miyata Eye Hospital from 2003 to 2006 were retrospectively analyzed. The study followed the tenets of the Declaration of Helsinki. All patients provided informed consent after receiving a full explanation of the nature and possible consequences of the study.

\section{Inclusion Criteria}

The diagnosis was based on clinical symptomatology, cerebrospinal fluid examination, and ophthalmoscopic and fluorescein angiography (FA) findings. Patients who met the Revised International Diagnostic Criteria for VKH disease ${ }^{16}$ as either complete VKH disease or incomplete VKH disease were included in this study. Further inclusion criteria were the following: (1) patients with an initial acute inflammatory episode of VKH disease who had an IA performed before systemic therapy was introduced, and (2) patients with an interval of less than 1 month between onset of symptoms and an IA examination.

\section{IA Procedure}

A standard IA protocol to analyze choroiditis designed and reported earlier ${ }^{6}$ was used. In Tokyo Medical and Dental University Hospital Ophthalmology Department, a Topcon TRC-50IA fundus camera coupled to an analogical video system was used, and images were later digitalized using a commercially available video digitalizing system (WinTVPVR2). In Miyata Eye Hospital, a Kowa VX-10i fundus camera that was coupled to a digitalizing system (VK-2) was used. The angiographic procedure comprises three main phases. The early phase of up to 2-3 min shows superimposed retinal and choroidal large vessels and physiological incipient exudation of the dye through the choriocapillaris into the choroidal stroma. The intermediate phase lasts about 10 min and shows maximum choroidal stromal background ICG fluorescence. The late phase, about 20-28 min or more, depending on fundus pigmentation, shows washout of the dye from the general circulation with the large choroidal vessels appearing dark against the background stromal fluorescence.

\section{IA Signs}

We chose to evaluate IA signs that were estimated to be most consistent as well as most useful and easy to evaluate and record for the initial evaluation of VKH disease, as previously described. ${ }^{13,14}$ Major IA signs, (1) early choroidal perfusion inhomogeneity, which is the same as disturbance/ delay in early choriocapillaris circulation; (2) early hyperfluorescent stromal vessels; (3) hypofluorescent dark dots (HDDs) seen in the intermediate and late phases; (4) fuzzy or lost vascular patterns of large stromal vessels seen in the intermediate to late angiographic phases; (5) disc hyperfluorescence; and (6) diffuse late choroidal hyperfluorescence, were analyzed and correlated with clinical findings, fundus pictures, FA findings, and optical coherence tomography (OCT) findings in some patients. Decimal visual acuity data were converted to logarithm of the minimum angle of resolution equivalents for statistical analysis.

\section{Results}

Ten patients from the two centers fulfilled the inclusion criteria. Patient data and clinical findings at presentation are shown in Table 1. There were five men and five women aged between 21 and 79 years (mean $47.2 \pm 17.5$ years). Cerebrospinal fluid pleocytosis and papillitis were seen in 
all cases. Mean best-corrected visual acuity (20 eyes) at presentation was $0.72(0.2-1.5)$. Anterior segment examination showed anterior uveitis in nine of ten patients (18 of 20 eyes), and this uveitis was typically granulomatous except in one patient. Fundus examination showed bilateral papillitis in all patients and multiple serous retinal detachments in 18 of 20 eyes. FA showed disc hyperfluorescence in all patients and subretinal fluid in 18 of 20 eyes. In four of ten patients OCT was available at the acute stage and showed serous retinal detachments in all patients.

IA findings at presentation are shown in Table 2. Choroidal perfusion inhomogeneity (Fig. 1) was seen in six of ten patients presenting some degree of choroidal filling delay in the early angiographic phase. The results are expressed for each patient because one eye of each patient had to be chosen for the early frames. Early hyperfluorescent stromal vessels (Fig. 2A, B) were present in all cases. HDDs (Fig. 3) were also present in all cases. A fuzzy or lost vascular pattern of large stromal vessels in the intermediate to late phase (Fig. 4) was also very consistent and present in all cases. Disc hyperfluorescence (Fig. 5A, B) was seen in nine of ten cases (18 of 20 eyes). Diffuse late choroidal hyperfluorescence (Fig. 6) was found in all patients. The most consistent findings, present in all cases, were early hyperfluorescent stromal vessels, HDDs, fuzzy or lost vascular pattern of large stromal vessels in the intermediate to late phase, and diffuse late choroidal hyperfluorescence.

\section{Discussion}

Among the IA signs looked for in VKH disease, early hyperfluorescent stromal vessels, HDDs, fuzzy or lost pattern of large stromal vessels in the intermediate to late angiographic phase, and disc hyperfluorescence were most consistent and therefore represent useful parameters for evaluating choroidal inflammatory involvement in Japanese patients. This set of universally found signs makes IA a reliable and precise method to assess and follow VKH patients. Analyzing these signs in chronologic order following the angiographic time sequence, the detailed findings can be summarized as follows with some physiopathological explanations.

Choroidal perfusion inhomogeneity (Fig. 1) has been described by most reports on IA in VKH disease and put forward as an important and specific sign. ${ }^{9-11}$ We found, however, that although this was sometimes a very clear sign when present, it was less consistent than other IA signs, as well as rather nonspecific and difficult to evaluate and quantify.

Early hyperfluorescent stromal vessels (Fig. 2A, B), which indicate severe choroidal stromal inflammatory vasculopathy, are a sign that appears in the early angiographic phase (up to $3 \mathrm{~min}$ ). In later phases, it gives way to diffuse late hyperfluorescence. This sign was easy to evaluate and the number of early hyperfluorescent vessel segments could even be counted, making it a quantitative or at least a semiquantitative parameter. 
Table 2. IA findings of patients

\begin{tabular}{|c|c|c|c|c|c|c|c|c|c|c|c|}
\hline \multirow[b]{2}{*}{ IA findings } & \multicolumn{10}{|c|}{ Patient } & \multirow{2}{*}{$\begin{array}{c}\text { Present } \\
(\%)\end{array}$} \\
\hline & 1 & 2 & 3 & 4 & 5 & 6 & 7 & 8 & 9 & 10 & \\
\hline Choroidal perfusion inhomogeneity $^{\mathrm{a}}$ & + & + & - & - & - & - & + & + & + & + & 60 \\
\hline Early hyperfluorescent stromal vessels ${ }^{\mathrm{b}}$ & $+/+$ & $+/+$ & $+/+$ & $+/+$ & $+/+$ & $+/+$ & $+/+$ & $+/+$ & $+/+$ & $+/+$ & 100 \\
\hline Hypofluorescent dark dots ${ }^{\mathrm{b}}$ & $+/+$ & $+/+$ & $+/+$ & $+/+$ & $+/+$ & $+/+$ & $+/+$ & $+/+$ & $+/+$ & $+/+$ & 100 \\
\hline Fuzzy or lost pattern of large stromal vessels ${ }^{b}$ & $+/+$ & $+/+$ & $+/+$ & $+/+$ & $+/+$ & $+/+$ & $+/+$ & $+/+$ & $+/+$ & $+/+$ & 100 \\
\hline Disc hyperfluorescence ${ }^{\mathrm{b}}$ & $+/+$ & $+/+$ & $+/+$ & $+/+$ & $+/+$ & $+/+$ & $+/+$ & $+/+$ & $+/+$ & $-1-$ & 90 \\
\hline Diffuse late choroidal hyperfluorescence ${ }^{b}$ & $+/+$ & $+/+$ & $+/+$ & $+/+$ & $+/+$ & $+/+$ & $+/+$ & $+/+$ & $+/+$ & $+/+$ & 100 \\
\hline
\end{tabular}

${ }^{a}$ In each patient (rather than in each eye) as one eye had to be chosen for the early frames.

${ }^{\mathrm{b}}$ Right eye/left eye.

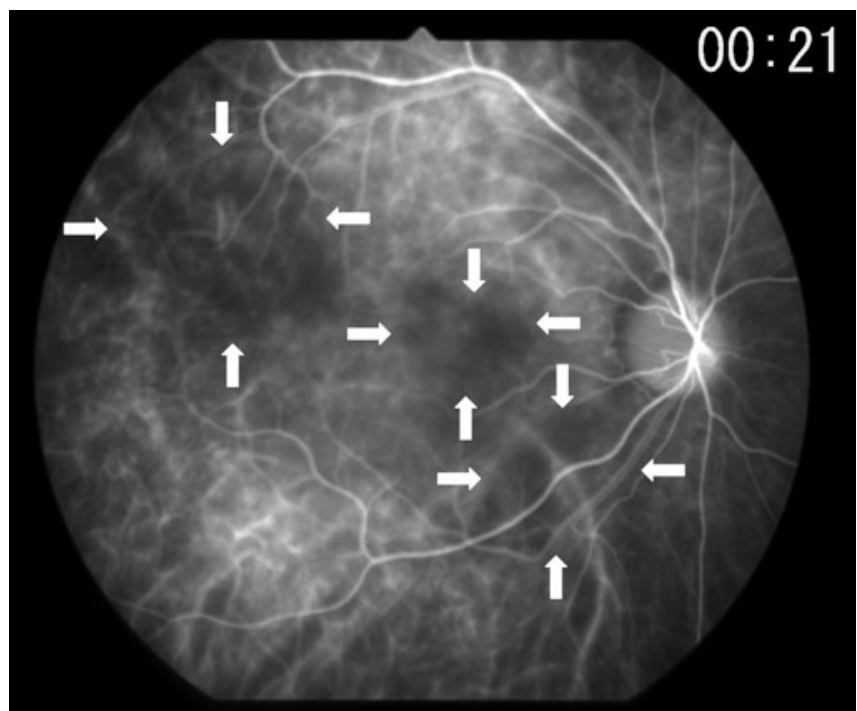

Figure 1. Choroidal perfusion inhomogeneity (patient 10). White arrows show choriocapillary hypoperfusion. This sign is found inconsistently and is difficult to standardize.

Hypofluorescent dark dots (HDDs) (Fig. 3), which are thought to correspond to choroidal granulomas, were the most consistent, most readily recordable angiographic sign, allowing also the assessment of choroidal inflammatory activity in a semi-quantitative way. HDDs can be evaluated by using an arbitrary semi-quantitative score from 0 to 4 based on the extension and number of dots, as described earlier. ${ }^{17}$

Fuzzy or lost vascular pattern of large stromal vessels (Fig. 4) indicate diffuse inflammatory vasculopathy of stromal vessels. This sign should be looked for in the intermediate to late phase, and it gives way in the late phase to diffuse stromal hyperfluorescence. In case of severe inflammation, the usual pattern of choroidal vessels can even become completely lost, preventing identification of individual vessels.

The optic disc usually remains dark and nonfluorescent in normal IA. Disc hyperfluorescence (Fig. 5A, B), rarely seen on IA, indicates severe papillitis with a blood-ocular barrier rupture sufficiently important to allow leakage of the macromolecular ICG-protein complex from disc capil- laries. Disc hyperfluorescence indicates very severe disease and usually regresses rapidly after introduction of initial induction therapy. It is therefore a good sign for evaluating the initial inflammatory activity of the disease and its response to therapy. ${ }^{13}$

Diffuse late choroidal hyperfluorescence (Fig. 6), although a consistent IA sign found in all our patients, is difficult to evaluate. The appearance of this sign is too dependent on technical factors such as the quantity of gain used. It is difficult to record and not sufficiently precise to be used in both assessment and follow-up of choroidal involvement.

In the present work, our aim was to study whether the IA signs previously identified and systematized could be applied to Japanese VKH patients and could be considered universally acceptable. We chose to evaluate IA signs that had been established as the most consistent as well as the most useful and easy to evaluate in previously published reports. ${ }^{6,13,14}$ All examined patients showed extensive choroidal involvement even at a very early stage of the disease.

As stated earlier, the diagnosis of VKH disease is essentially clinical, based on bilaterality, multiple serous retinal detachments, and papillitis, together with prodromal systemic symptoms such as malaise and headaches as well as lymphomonocytic pleocytosis of the cerebrospinal fluid. FA reveals better evidence of these clinical signs, showing disc hyperfluorescence and allowing exact delineation of the serous retinal detachments with their leaking points. Similarly, OCT allows clear depiction of the height and extension of serous retinal detachments in the macular area, but apart from the high-quality images obtained, it provides no essential additional information that is not already available by clinical examination. ${ }^{18}$ The explanation for this is that clinical signs, FA, and OCT, all give merely information on secondary inflammatory events that occur when inflammation is spilling over from the choroid. IA has been found to be particularly useful for early disease detection when no secondary spill-over inflammation is present as yet. ${ }^{19}$ The advantage of IA is that it allows localized choroidal inflammation to be detected when it has not yet affected adjacent structures and become clinically apparent. It has to be kept in mind that in VKH disease the primary and main inflammatory events take place and originate within the choroid, where the target of the autoimmune inflammatory reaction is situated. 

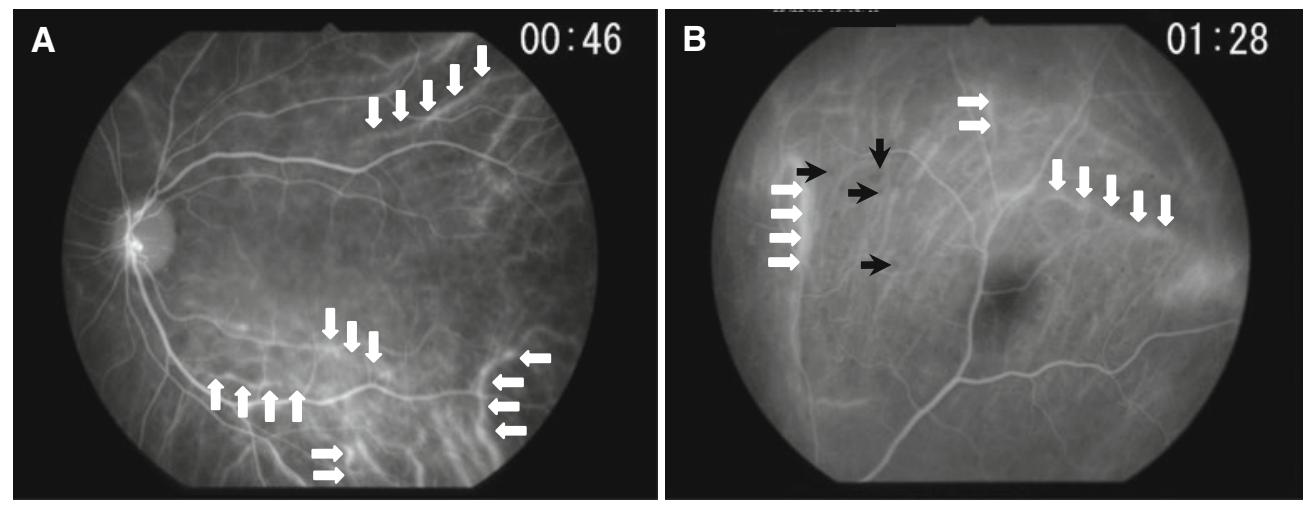

Figure 2A, B. Early hyperfluorescent stromal vessels (patient 10), a consistent indocyanine green angiography (IA) sign found in all our patients. A Some of the hyperfluorescent stromal vessels are outlined by the white arrows. Most stromal vessels are hyperfluorescent between the horizontal arrows in the lower part of the picture. B Early hyperfluorescent stromal vessels in early phase of angiography (up to 3 min); some are outlined by the white arrows. Numerous hypofluorescent dark dots start to become visible; some are indicated by the black arrows.

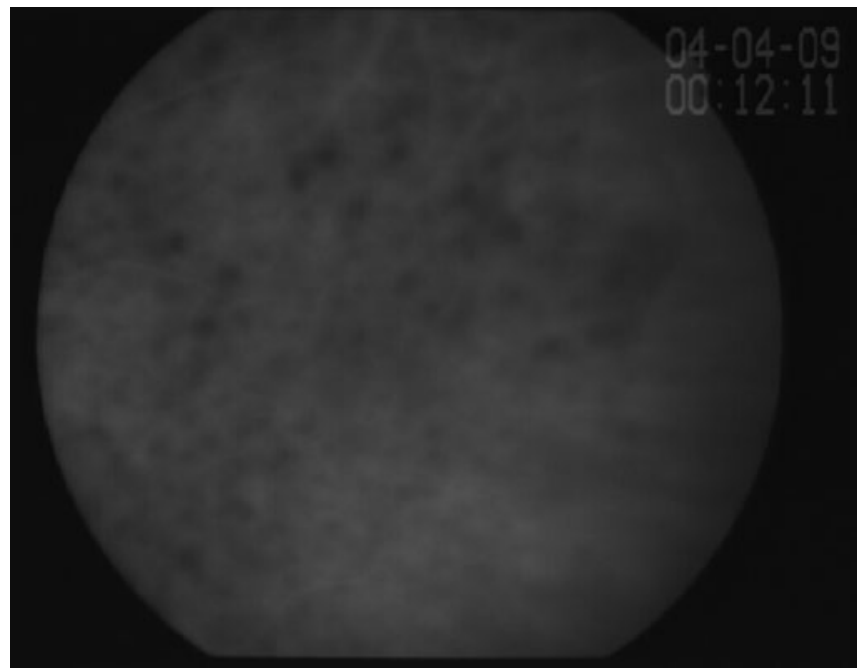

Figure 3. Hypofluorescent dark dots (HDDs) in late angiographic phase (patient 4). Numerous HDDs are seen in the late angiographic phase. Persistence of HDDs in the late angiographic phase indicates that the choroidal inflammatory foci (granulomas) occupy the whole thickness of the choroid and thus do not allow the IA dye to diffuse to these areas. Note also that no pattern of stromal vessels can be recognized any more. In this case the stromal vessels are so fuzzy that their network cannot be identified any more.

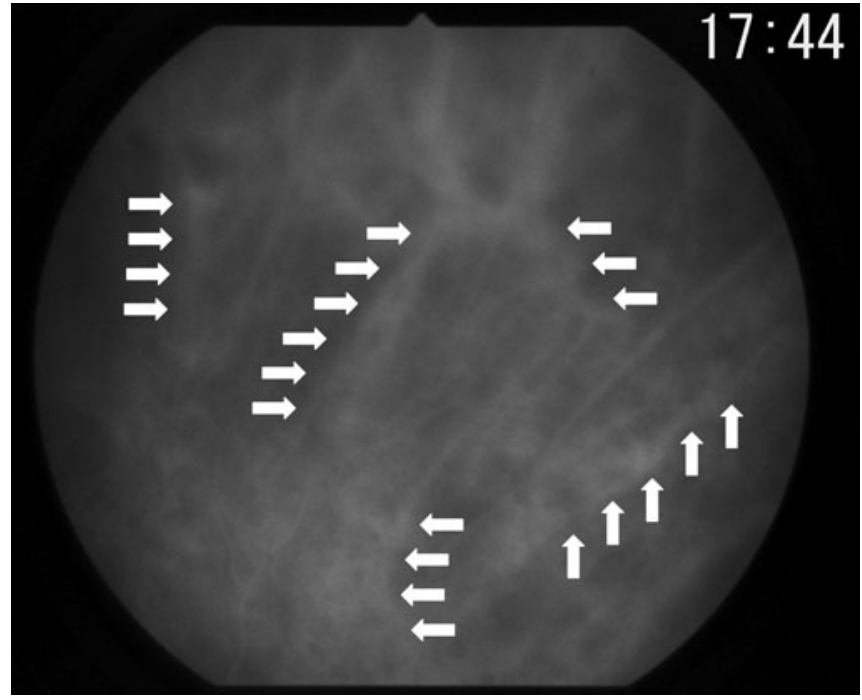

Figure 4. Fuzzy or lost pattern of large stromal vessels (patient 10). The stromal vascular pattern is still recognizable but the choroidal vessels have a fuzzy appearance (white arrows).
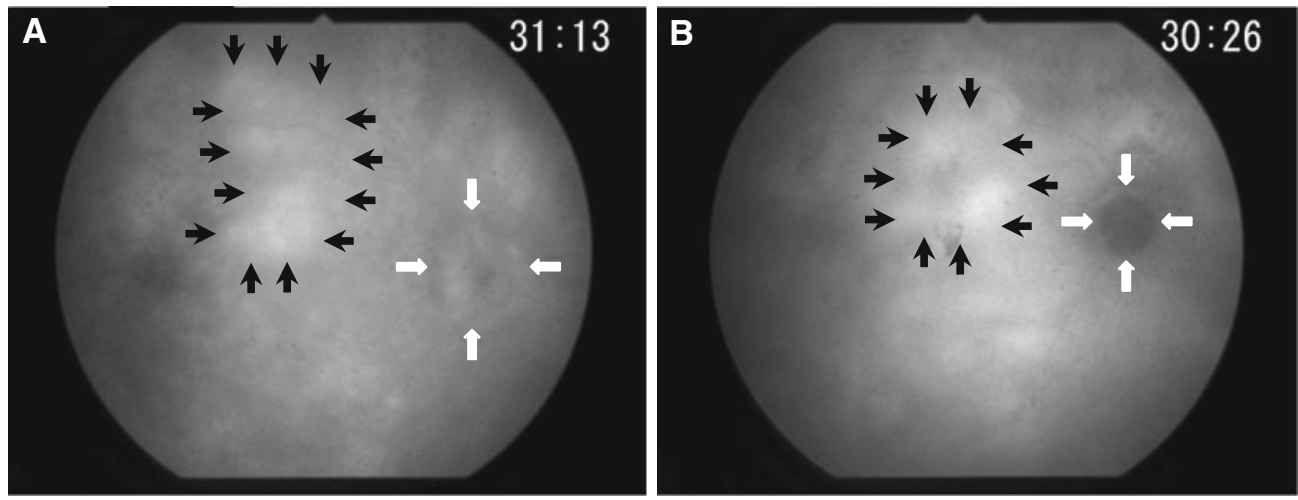

Figure 5A, B. Disc hyperfluorescence (patient 9). The optic disc usually remains hypofluorescent unless severe inflammation is present. A Latephase frame showing an extremely hyperfluorescent disc (white arrows). The black arrows delineate a zone of diffuse late choroidal hyperfluorescence. B Same case as in $\mathbf{A}$ after 1 month of treatment: disc hyperfluorescence has completely regressed (white arrows), whereas diffuse late hyperfluorescence is still present (black arrows). 


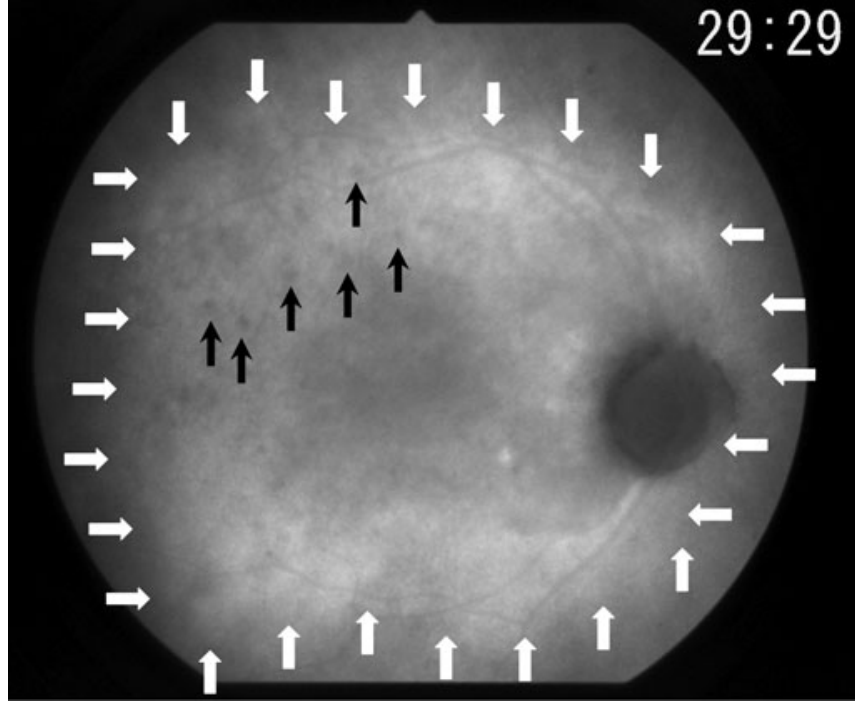

Figure 6. Diffuse late choroidal hyperfluorescence (patient 10). The areas of late diffuse hyperfluorescence are delineated by the white arrows. Within these areas numerous HDDs are visible (black arrows) Diffuse late choroidal hyperfluorescence is difficult to standardize and is less practical to use as a follow-up sign.

\section{References}

1. Sugiura S. Vogt-Koyanagi-Harada disease. Jpn J Ophthalmol 1978;22:9-35.

2. Moorthy RS, Inomata H, Rao NA. Vogt-Koyanagi-Harada syndrome. Surv Ophthalmol 1995;39:265-292.

3. Gocho K, Kondo I, Yamaki K. Identification of autoreactive T cells in Vogt-Koyanagi-Harada disease. Invest Ophthalmol Vis Sci 2001;42:2004-2009.

4. Damico FM, Cunha-Neto E, Goldberg AC, et al. T-cell recognition and cytokine profile induced by melanocyte epitopes in patients with HLA-DRB1*0405-positive and -negative Vogt-KoyanagiHarada uveitis. Invest Ophthalmol Vis Sci 2005;46:2465-2471.

5. Sugita S, Takase H, Taguchi C, et al. Ocular infiltrating CD4+ T cells from patients with Vogt-Koyanagi-Harada disease recognize human melanocyte antigens. Invest Ophthalmol Vis Sci 2006; 47:2547-2554.
6. Herbort CP, LeHoang P, Guex-Crosier Y. Schematic interpretation of indocyanine green angiography in posterior uveitis using a standard protocol. Ophthalmology 1998;105:432-440.

7. Bouchenaki N, Herbort CP. Stromal choroiditis. In: Pleyer U, Mondino B, editors. Essentials in ophthalmology: uveitis and immunological disorders. Berlin, Heidelberg, New York: Springer; 2004. p. 234-253.

8. Bouchenaki N, Cimino L, Auer C, Tran VT, Herbort CP. Assessment and classification of choroidal vasculitis in posterior uveitis using indocyanine green angiography. Klin Monatsbl Augenheilk 2002:219:243-249.

9. Yuzawa M, Kawamura A, Matsui M. Indocyanine green videoangiographic findings in Harada's disease. Jpn J Ophthalmol 1993;37:456-466.

10. Oshima Y, Harino S, Hara Y, Tano Y. Indocyanine green angiographic findings in Vogt-Koyanagi-Harada disease. Am J Ophthalmol 1996;122:58-66.

11. Okada AA, Mizusawa T, Sakai J, Usui M. Videofunduscopy and videoangiography using the scanning laser ophthalmoscope in Vogt-Koyanagi-Harada syndrome. Br J Ophthalmol 1998;82: 1175-1181.

12. Kohno T, Miki T, Shiraki K, et al. Subtraction ICG angiography in Harada's disease. Br J Ophthalmol 1999;83:822-833.

13. Bouchenaki N, Herbort CP. The contribution of indocyanine green angiography to the appraisal and management of Vogt-KoyanagiHarada. Ophthalmology 2001;108:54-64.

14. Herbort CP, Mantovani A, Bouchenaki N. Indocyanine green angiography in Vogt-Koyanagi-Harada disease: angiographic signs and utility in patient follow-up. Int Ophthalmol 2007;27:173182

15. Kawaguchi T, Horie S, Bouchenaki N, et al. Suboptimal therapy controls clinically apparent disease but not subclinical progression of Vogt-Koyanagi-Harada disease. Int Ophthalmol 2010;30: 41-50.

16. Read RW, Holland GN, Rao NA, et al. Revised diagnostic criteria for Vogt-Koyanagi-Harada disease: report of an international committee on nomenclature. Am J Ophthalmol 2001;131: 647-652.

17. Altan-Yaycioglu R, Akova YA, Akca S, Yilmaz G. Inflammation of the posterior uvea: findings on fundus fluorescein and indocyanine green angiography. Ocul Immunol Inflamm 2006;14:171179.

18. Mantovani A, Resta A, Herbort CP, et al. Work-up, diagnosis and management of acute Vogt-Koyanagi-Harada disease: a case of acute myopization with granulomatous uveitis. Int Ophthalmol 2007;27:105-115.

19. Howe L, Stanford M, Graham E, Marshall J. Indocyanine green angiography in inflammatory eye diseases. Eye 1998;12:761-767. 